\title{
Exercises and sports participation in healthy older adults with intellectual disability-A pilot study
}

\author{
Eli Carmeli ${ }^{1 *}$, Joav Merrick ${ }^{2}$, Bita Imam ${ }^{3}$, Ran Levy ${ }^{4}$ \\ ${ }^{1}$ Department of Physical Therapy, Neve Ram Residential Care Center, Rechasim, Israel; \\ ${ }^{*}$ Corresponding Author: elie@post.tau.ac.il \\ ${ }^{2}$ National Institute of Child Health and Human Development, Health Services, Office of the Medical Director, Division for Mental \\ Retardation, Ministry of Social Affairs, Jerusalem, Israel; jmerrick@,zahav.net.il \\ ${ }^{3}$ Rehabilitation Research Lab, GF Strong Rehabilitation Centre, Vancouver, Canada; bita imam@yahoo.com \\ ${ }^{4}$ Neve Ram Residential Care Center, Rechasim, Israel; ran@neve-ram.org.il
}

Received 31 July 2012; revised 27 August 2012; accepted 4 September 2012

\section{ABSTRACT}

Background: Many adults with intellectual disabilities (ID) have low physical activity (PA). Lack of PA is a risk factor for metabolic and cardiovascular diseases, and some types of cancer. The objective of this study was to investigate the rate of PA in healthy older adults with ID. Methods: A cross-sectional analysis of baseline data. Participants were caregivers of persons with ID who were over 50 years old and living in a residential care center. A PA questionnaire and a daily rapport form were used to gather information about the type and rate of daily PA of persons with ID for 12 consecutive days. The Katz Index of Independence in Activities of Daily Living (ADL) was used to assess functional dependency. Results: Healthy older adults with ID do not engage in adequate regular PA as recommended by the US Department of Health and Human Services. Conclusion: The low participation in PA for persons with ID may be related to lack of appreciation of the benefits of PA, lack of support from their caregivers and difficulty finding experienced personnel to train them.

Keywords: Aging; Intellectual Disability; Physical Activity

\section{INTRODUCTION}

Health prevention and promotion in term of physical activity (PA) among aged individuals with intellectual disability (ID) has received little attention from policy makers and health professionals in the public health and welfare authorities.

A large body of evidence regarding physical activity in persons of ID provided a useful information regarding the concept of physical activity versus exercise and sports, and regarding the limitations of the use of questionnaires [1-6]. However, there are no data whether persons with ID meet the recommendations for PA made by US department of health and human services [7]. The recommendations are designed to provide information and guidance on the types and amounts of PA that provide substantial short-and-long term health benefits. The main idea behind the recommendations is that regular PA over months and years plays a key role in manifestation of long-term health benefits such as disease prevention [8], and more specifically improving the quality of life of people with mental disorders [9]. According the guidelines "for substantial health benefits, adults should do at least 150 minutes (2 hours and 30 minutes) a week of moderate-intensity, or 75 minutes (1 hour and 15 minutes) a week of vigorous-intensity aerobic physical activity, or an equivalent combination of moderate- and vigorous-intensity aerobic activity. Aerobic activity should be performed in episodes of at least 10 minutes, and preferably, it should be spread throughout the week".

The majority of aged people with intellectual disabilities in the Israel live at residential care centers. People with ID living in residential care centers benefit from social and psychological support, and they participate in a more active life style on a regular basis [10]. Although the life expectancy of this population nowadays is increasing over time and people with ID live much longer than they did in the past, yet, their ageing process start as early as 50 years of age which is much earlier than for others in the general population [11].

Promoting physical exercise in aged people with ID is particularly important since these individuals are prone to high risk of chronic morbidity and sedentary life style resulted in overweight, difficulties in active daily living (ADL), frequent hospitalization, frailty, falls and mortal- 
ity [12]. However, for persons with ID, participation in regular PA is considered somewhat incomprehensible, challenging and complex due to environmental barriers, inappropriate facility, lack of motivation, and consistency, and poor retention [13]. Exploring the rate of PA engagement it is essential since they portray special needs.

Therefore, the main objective of this study was to evaluate level of ADL independency, to investigate the rate of participation in $\mathrm{PA}$, the type of $\mathrm{PA}$, and to explore the external interferences associated with not doing PA.

\section{MATERIALS AND METHODS}

This observational pilot study was based on crosssectional analyses of baseline data through 12 consecutive days. Persons with ID were recruited from NeveRam residential care center through a convenience sampling trial entitled "screening for a weekly PA".

\subsection{Participants}

A convenience sample comprised persons with "unspecified ID" who were the permanent residents of the care center, and aged 50 or older. Therefore, to be eligible, the ID participants had to have unspecified moderate ID (e.g., fragile X and Down syndrome were excluded), be able to walk at least 100 meters independently, and have no acute heart or pulmonary disease, acute musculoskeletal disease, malignancy, or other medical contraindications for engaging in PA.

\subsection{Measurements}

A PA questionnaire and a daily rapport form were used to gather information regarding the rate and type of PA. These forms were reported by the in house caregivers. Engagement in PA is defined as involvement in structural physical training that involves skeletal muscle contraction and energy consumption (e.g., floor exercises, treadmill), and sports activity (e.g., outdoor walking, biking, swimming, football) for at least 15 consecutive minutes.

The PA questionnaire was divided into three parts. The first two parts were completed by the study's Principal Investigator. Part I, "standardized demographic questions", captures information about date of birth, gender, level of mental retardation, and years living at the center. Part II, "standardized health questions", gathers data regarding Body Mass Index (weight $(\mathrm{kg}) /$ height $^{2}$ ( meter $^{2}$ ), clinical manifestations, and medications used in the past four weeks. Part III, "bi-weekly PA questionnaire", rates the amount of participation in PA in a Likert-type scale system: 1 (none); 2 (once a week); 3 (2 - 3 times a week); 4 (4 - 5 times a week); and 5 (6 - 7 times a week). In addition, part III gathers information about the duration in minutes and types of PA, such as outdoor walking, biking, swimming, ball games, horse riding, bowling, etc. Part III was completed by the caregivers. The caregivers were very familiar with all the residents at the residential care centers. Each caregiver was first informed about the voluntary nature of his/her participation. The caregivers were instructed in details on how to collect and record data. To ensure valid method for assessing physical activity each caregiver received an explicated description of what is truly and justly considered PA.

Data were gathered twice for six consecutive days (for a total of 12 working days, Saturdays excluded). It took approximately five minutes for the caregivers to complete Part III.

The content validity of Part III of the questionnaire was tested by 3 independent experts in ID and PA for aging people. Each item was assessed for relevancy, ease for the participants to understand (e.g., language, vocabulary) for evaluating the "what" and "how" PA they engaged on a regular basis.

To assess the status of functional independency of persons with ID, the six-item Katz Index of Independence in Activities of Daily Living (ADL) was used [14]. The Katz ADL scale assesses basic personal ADL and ranks independency of performance in six functions (bathing, dressing, going to the toilet, transferring from bed to chair, continence, and feeding). Dependency in each personal activity was evaluated and a summary score ranging from 0 (independent in all activities) to 6 (dependent in all activities) was obtained.

Ethical approval for the study was granted by the ethics committee from the Israeli Office of the Medical Director, Health and Welfare Services for the Division of Mental Retardation, by the IRB of Neve Ram Institute. Oral consent was obtained from the all participants as well as informed, written consent from their guardians.

\subsection{Statistical Analysis}

Statistical analysis was performed using SAS (Statistical Analysis System) version 9.0 for Windows. Means and standard deviations (SDs) of the variables were calculated. The significance level was set at 5\%. Average daily activity was calculated for each participant.

\section{RESULTS}

All recruited caregivers from the residential care center agreed to participate $(n=7)$. Of 33 recruited ID individuals, 21 were enrolled in the study. The mean \pm SD age of the sample was $55.79 \pm 3.23(56.46 \pm 3.3$ for females, and $55.12 \pm 3.17$ for males). The sample was predominantly female (59\%). Quarter of the sample had mild-moderate ID, while the rest were diagnosed with moderate or severe level ID (Table 1). 
Table 1. Demographics and clinical manifestations of the sample $(\mathrm{n}=21)$.

\begin{tabular}{|c|c|c|}
\hline & Value & Percentage \\
\hline \multicolumn{3}{|l|}{ Age $($ mean \pm SD $)$} \\
\hline Males & 55.12 & \\
\hline Females & 56.46 & \\
\hline \multicolumn{3}{|l|}{ Gender (n) } \\
\hline Males & 8 & 41 \\
\hline Females & 13 & 59 \\
\hline \multicolumn{3}{|l|}{ Level of ID (n) } \\
\hline Mild-moderate & 5 & 24 \\
\hline Moderate & 7 & 35 \\
\hline Moderate-severe & 9 & 51 \\
\hline \multicolumn{3}{|l|}{ Years living in the center (n) } \\
\hline Less than 5 & 3 & Less than 5 \\
\hline Less than 10 & 5 & Less than 10 \\
\hline More than 11 & 13 & More than 11 \\
\hline \multicolumn{3}{|l|}{$\mathrm{BMI}($ mean $\pm \mathrm{SD})$} \\
\hline Males & $25.2 \pm 2.3$ & \\
\hline Females & $26.8 \pm 2.7$ & \\
\hline \multicolumn{3}{|l|}{ Clinical manifestations } \\
\hline Cardiac disease & & 20 \\
\hline Hypertension & & 60 \\
\hline Diabetes & & 2 \\
\hline $\begin{array}{l}\text { Respiratory disease } \\
\text { (COPD, asthma) }\end{array}$ & & 4 \\
\hline $\begin{array}{l}\text { Neoplastic disease } \\
\text { (benign \& malignant tumors) }\end{array}$ & & - \\
\hline $\begin{array}{l}\text { Renal disease } \\
\text { (insufficiency and chronic UTI) }\end{array}$ & & 2 \\
\hline Vascular disease & & 4 \\
\hline $\begin{array}{l}\text { GI diseases (gastritis, duodenal } \\
\text { ulcer, chronic constipation) }\end{array}$ & & 8 \\
\hline \multicolumn{3}{|l|}{ Medications (no/daily) } \\
\hline $1-2$ & & 25 \\
\hline $3-4$ & & 40 \\
\hline$>5$ & & 35 \\
\hline \multicolumn{3}{|l|}{ Katz ADL score ${ }^{*}$} \\
\hline Males & & 4.8 \\
\hline Females & & 4.2 \\
\hline
\end{tabular}

ADL: Activities of Daily Living; BMI: Body Mass Index (body weight (kg)/ height ${ }^{2}\left(\mathrm{~m}^{2}\right)$ ); COPD: Chronic Obstructive Pulmonary Disease; GI: Gastro intestine; UTI: Upper Tract Infection; ${ }^{*}$ Katz score: Independence: (1 point) no supervision, direction or personal assistance; Dependence: ( 0 point) with supervision, direction, personal assistance or total care; Total score: $6=$ highest (independent); $0=$ lowest (very dependent).

\subsection{The Katz Index ADL}

Approximately $40 \%$ of the participants with ID had difficulty in bathing and going to the toilet and $25 \%$ had difficulty in grooming.

\subsection{PA Questionnaire}

The Fleiss' Kappa was $0.778(\mathrm{p}<0.005)$. This degree of agreement is statistically significant. The prevalence, type, duration and frequency of reported participation in PA during 12 days revealed a low level of participation. The average duration of weekly PA recorded by the caregivers was less than 100 minutes. The most prevalent activity was "walking". The data revealed that $75 \%$ of the participants engaged in outdoor walking at least 2 - 3 times a week for a total of 30 - 75 minutes a week, and a few engaged once a week in swimming (8\%), general exercises (14\%) and other activities such as ball games (4\%), yard work (4\%) and dance (8\%) (Table 2). The PAs were mainly performed in the morning $(70 \%)$, either with supervision of caregivers $(60 \%)$, physical educators/athletic trainers $(20 \%)$ or physical therapists $(20 \%)$.

\section{DISCUSSION}

Engagement in PA is essential for older people, especially for those with ID. Regular engagement in PA has many health benefits, including preventing chronic conditions and maintaining mobility and community independence as recommended by the US Department of Health and Human Services [7]. According to the guidelines, "older people should do 120 minutes of moderate intensity aerobic and 30 minutes of moderate intensity anaerobic activity a week in order to maintain or improve physical function and independent living; improve social interactions, quality of life, and reduce depression; build and maintain healthy bones, muscles and joints, reduce the risk of injuries from falls; and reduce the risk of heart disease, stroke, high blood pressure, type II diabetes, and some types of cancer". To the authors' knowledge, no other studies have investigated whether older persons with ID who live in residential care facilities meet the recommended PA guidelines. There are no specific guidelines for healthcare professionals regarding how physical exercises should be done for older persons with ID. Moreover, little is known about the type and pattern (i.e., intensity, duration, frequency) of physical exercises that person with ID use and the barriers to accessing certain exercises; although efforts are currently being made to explore these questions.

This pilot study was used to assess the feasibility of the study design and in particularly to inquire the degree of cooperation by the participants, the sincerity of their caregivers to record the questionnaire, and to detect any potential obstacles.

The results of this study demonstrated that a fair proportion of persons with ID living in residential care centers do not engage in PA up to the recommended guidelines despite the well-structured and organized environment of the facilities in which they live. The average 
Table 2. Results of the PA questionnaire.

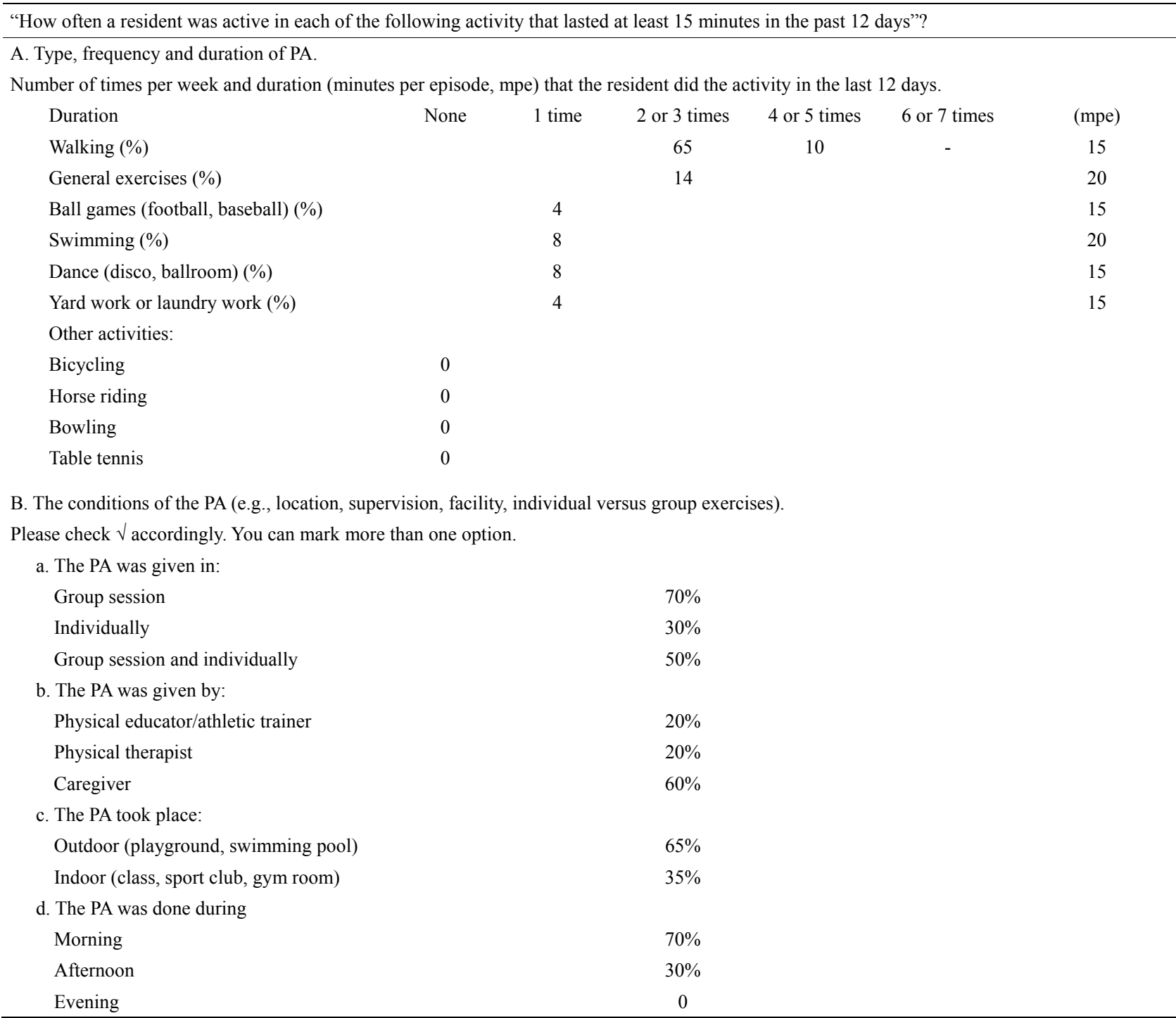

duration of weekly PA as reported by the caregivers was less than 100 minutes a week, which is below the recommended guidelines given by US Department of Health and Human Services. Inadequate involvement in regular PA among ID individuals may contribute to displaying premature age-related changes in their health [15]. Although the present study sheds light on the quantity and types of PA that persons with ID engage in, the underlying reasons for the low rate of PA remain unrevealed and thus warrant further studies. There is a need to provide specialized healthcare to the aging population of adults with ID who are at high risk for developing medical conditions [16]. A specialized healthcare to this unique population is essential since they are not fully aware to the short and long term benefits of primary and secondary prevention.

Older adults with ID are characterized as having a passive lifestyle, sedentary behaviors, poor motor perceptions, poor self-efficacy, and a high fear of falling [17]. As they age, persons with ID may adapt to this "lifestyle", and the desire or the need to be physically active may diminish over time. Thus it is possible that the lack of appreciation for PA is a consequence rather than a cause of an unmet need for PA. The possible barriers to participation in PA may be related to caregivers' support and the difficulty of finding experienced personnel to train them. As we instructed the caregivers on how to complete the PA questionnaire, we noticed that the caregivers did not value the benefits of PA enough, and it is possible that they do not provide related opportunities for their clients to engage in PA. Consistent with our observation, the low educational level of staff working with ID individuals in community residences has been recognized as a barrier to low PA among this group [18]. 
Therefore, there is a need to educate caregivers of older adults with ID on the benefits and the necessity of PA.

This study however has a number of limitations. The sample size was small and this limits the generalisability of the results and therefore it is hard to reflect the whole situation of older adults with ID. Yet, this was a pilot study which was found workable and practicable. Future study with more participates should be included to support the present investigation, as well as the rate of PA among aged-and-gender match participants without ID should be included as a control group.

The use of questionnaires filled out by caregivers to rate the PA of the persons with ID might be a potential source of bias if the caregivers provided answers in the light of satisfying the system by exaggerating the amount of the actual PA involvement; thus the accuracy of proxy report remains questionable. Assessing the intensity of PA in individuals with ID might not be feasible since the majority of the participants do not exert or invest enough effort during PA. Moreover, most of the ID residents were unwilling to participate in PA due to difficulties in understanding the importance of regular PA and the consequences of not doing PA. A method for assessing PA (e.g., pedometers, heart rate monitors) coupled with selfquestionnaire could enhance the validity of the study. The other limitation of this study is that we were compelled to exclude the most disabled people from the study; therefore the results probably underestimate the extent of the problem.

\section{CONCLUSION}

As individuals with ID continue to experience longer lives, the need to understand their aging and associated health conditions becomes more critical. The chronic disorders that develop in adults with ID have important implications for healthcare management of this aging population. Unfortunately, older adults with ID do not engage in adequate regular PA as recommended by the US Department of Health and Human Services. Lack of appreciation for PA by both persons with ID and their caregivers, and difficulty finding experienced personnel to train them, are possible barriers.

\section{REFERENCES}

[1] Hilgenkamp, T.I., van Wijck, R. and Evenhuis, H.M. (2012) Feasibility and reliability of physical fitness tests in older adults with intellectual disability: A pilot study. Journal of Intellectual Development and Disability, 37, 158-162. doi:10.3109/13668250.2012.681773

[2] Bartlo, P. and Klein, P.J. (2011) Physical activity benefits and needs in adults with intellectual disabilities: Systematic review of the literature. American Journal of Intellectual Development and Disability, 116, 220-232. doi:10.1352/1944-7558-116.3.220

[3] Pitetti, K.H., Beets, M.W. and Combs, C. (2009) Physical activity levels of children with intellectual disabilities during school. Medicine and Sciences and Sports Exercises, 41, 1580-1586. doi:10.1249/MSS.0b013e31819d4438

[4] Morey, M.C., Sloane, R., Pieper, C.F., Peterson, M.J., Pearson, M.P., Ekelund, C.C., Crowley, G.M., DemarkWahnefried, W., Snyder, D.C., Clipp, E.C. and Cohen, H.J. (2008) Effect of physical activity guidelines on physical function in older adults. Journal of American Geriatric Society, 56, 1873-1878. doi:10.1111/j.1532-5415.2008.01937.x

[5] Kruger, J., Buchner, D.M. and Prohaska, T.R. (2009) The prescribed amount of physical activity in randomized clinical trials in older adults. Gerontologist, 49, S100S107. doi:10.1093/geront/gnp075

[6] Temple, V.A. (2009) Factors associated with high levels of physical activity among adults with intellectual disability. International Journal of Rehabilitation and Research, 32, 89-92. doi:10.1097/MRR.0b013e328307f5a0

[7] (2008) US department of health and human services: Physical activity guidelines for Americans.

http://www.health.gov/PAGuidelines/guidelines/summary aspx

[8] Sattelmair, J.R., Pertman, J.H. and Forman, D.E. (2009) Effects of physical activity on cardiovascular and noncardiovascular outcomes in older adults. Clinical Geriatric Medicine, 25, 677-702. doi:10.1016/j.cger.2009.07.004

[9] Deslandes, A., Moraes, H., Ferreira, C., Veiga, H., Silveira, H., Mouta, R., Pompeu, F.A., Coutinho, E.S. and Laks, J. (2009) Exercise and mental health: Many reasons to move. Neuropsychobiology, 59, 191-198. doi: $10.1159 / 000223730$

[10] Merrick, J., Kandel, I., Lotan, M., Aspler, S., Fuchs, B.S. and Morad, M. (2010) National survey 2007 on medical services for persons with intellectual disability in residential care in Israel. International Journal of Adolescence and Medical Health, 22, 575-582. doi:10.1515/IJAMH.2010.22.4.575

[11] Lin, J.D., Wu, C.L., Lin, P.Y., Lin, L.P. and Chu, C.M. (2011) Early onset ageing and service preparation in people with intellectual disabilities: Institutional managers' perspective. Research and Developmental Disabilities, 32, 188-193. doi:10.1016/j.ridd.2010.09.018

[12] Carmeli, E., Merrick, J., Kessel, S., Masharawi, Y. and Carmeli, V. (2003) Elderly persons with intellectual disability: A study of clinical characteristics, functional status, and sensory capacity. Scientific World Journal, 28, 298-307. doi:10.1100/tsw.2003.24

[13] Temple, V.A. (2007) Barriers, enjoyment, and preference for physical activity among adults with intellectual disability. International Journal of Rehabilitation and Research, 30, 281-287. doi:10.1097/MRR.0b013e3282f144fb

[14] Katz, S., Ford, A.B., Moskowitz, R.W., Jackson, B.A. and Jaffe, M.W. (1963) Studies of illness in the aged. The index of ADL: A standardized measure of biological and 
psychosocial function. Journal of American Medical Association, 185, 914-919.

doi:10.1001/jama.1963.03060120024016

[15] Carmeli, E., Imam, B. and Merrick, J. (2012) The relationship of pre-sarcopenia (low muscle mass) and sarcopenia (loss of muscle strength) with functional decline in individuals with intellectual disability (ID). Archives of Gerontology and Geriatrics, 55, 181-185. doi:10.1016/j.archger.2011.06.032

[16] Bigby, C., Webber, R., Bowers, B. and McKenzie-Green, B. (2008) A survey of people with intellectual disabilities living in residential aged care facilities in Victoria. International Journal of Disability Research, 52, 404-414. doi:10.1111/j.1365-2788.2007.01040.x

[17] Carmeli, E., Bar-Chad, S., Lotan, M., Merrick, J. and Coleman, R. (2003) Five clinical tests to assess balance following ball exercises and treadmill training in adult persons with intellectual disability. Journal of Gerontology A: Biological Sciences of Medical Sciences, 58, 767-772. doi:10.1093/gerona/58.8.M767

[18] Elinder, L.S., Bergström, H., Hagberg, J., Wihlman, U. and Hagströmer, M. (2010) Promoting a healthy diet and physical activity in adults with intellectual disabilities living in community residences: Design and evaluation of a cluster-randomized intervention. BMC Public Health, 10, 761. doi: $10.1186 / 1471-2458-10-761$ 